\title{
Epidemiology and resistance phenotypes of carbapenemase-producing Klebsiella pneumoniae in Greece, 2014 to 2016
}

Irene Galani', Ilias Karaiskos², Irene Karantani³, Vassiliki Papoutsaki³, Sofia Maraki, Vassiliki Papaioannou ${ }^{5}$, Polyzo Kazila6,

Helen Tsorlini ${ }^{7}$, Nikoletta Charalampaki, Marina Toutouza9, Helen Vagiakou ${ }^{10}$, Konstantinos Pappas ${ }^{11}$, Anna Kyratsa ${ }^{12}$,

Konstantina Kontopoulou ${ }^{13}$, Olga Legga ${ }^{14}$, Efthymia Petinaki ${ }^{15}$, Helen Papadogeorgaki ${ }^{3}$, Efrosini Chinou ${ }^{16}$, Maria Souli ${ }^{1}$, Helen

Giamarellou $^{2}$, on behalf of the study collaborators ${ }^{17}$

1. Infectious Diseases Laboratory, 4th Department of Internal Medicine, National and Kapodistrian University of Athens, School of Medicine, Athens, Greece

2. 6th Department of Internal Medicine, Hygeia General Hospital, Athens, Greece

3. Microbiology Laboratory, Hygeia General Hospital, Athens, Greece

4. Department of Clinical Bacteriology, Parasitology, Zoonoses and Geographical Medicine, University Hospital of Heraklion, Heraklion, Greece

5. Microbiology Department, KAT Hospital, Athens, Greece

6. Cancer Hospital of Thessaloniki 'THEAGENEIO', Thessaloniki, Greece

7. Microbiological Laboratories, Bacteriology Department 'G. Papanikolaou' General Hospital of Thessaloniki, Thessaloniki, Greece

8. Department of Clinical Microbiology, Thriassio General Hospital, Elefsina, Athens, Greece

9. Department of Microbiology, Hippokration Athens General Hospital, Athens, Greece

10. Microbiology Laboratory, General Hospital of Athens 'G. Gennimatas', Athens, Greece

11. Athens Naval Hospital, Athens, Greece

12. Microbiology Laboratory, General Hospital of Corfu, Corfu, Greece

13. Department of Microbiology, General Hospital of Thessaloniki ‘G. Gennimatas', Thessaloniki, Greece

14. Department of Microbiology, General Hospital of Lamia, Lamia, Greece

15. Department of Microbiology, University Hospital of Larissa, Larissa, Greece

16. Department of Microbiology, St Savvas Cancer Hospital, Athens, Greece

17. The study collaborators are acknowledged at the end of the article

Correspondence: Irene Galani (egalani@med.uoa.gr)

Galani Irene, Karaiskos Ilias, Karantani Irene, Papoutsaki Vassiliki, Maraki Sofia, Papaioannou Vassiliki, Kazila Polyzo, Tsorlini Helen, Charalampaki Nikoletta, Toutouza Marina, Vagiakou Helen, Pappas Konstantinos, Kyratsa Anna, Kontopoulou Konstantina, Legga Olga, Petinaki Efthymia, Papadogeorgaki Helen, Chinou Efrosini, Souli Maria, Giamarellou Helen, on behalf of the study collaborators. Epidemiology and resistance phenotypes of carbapenemase-producing Klebsiella pneumoniae in Greece, 2014 to 2016. Euro Surveill. 2018;23(31):pii=1700775. https://doi.org/10.2807/1560-7917.ES.2018.23.30.1700775

Background and aim: A multicentre nationwide surveillance study was conducted in Greek hospitals to evaluate epidemiology of carbapenemase-producing Klebsiella pneumoniae clinical isolates, and their susceptibilities to last-line antibiotics. Methods: Minimum inhibitory concentrations (MICs) were evaluated by Etest, colistin MICs were also evaluated by broth microdilution SensiTest (now known as ComASP) Colistin. Carbapenemase genes were detected by PCR. Clonal relatedness was assessed by PFGE. Isolates were prospectively collected between November 2014 and April 2016, from 15 hospitals. Results: Among 394 isolates, $K$. pneumoniae carbepenemase (KPC) remained the most prevalent carbapenemase $(66.5 \%)$. NDM was the second most prevalent (13.7\%), identified in 12 hospitals, followed by VIM (8.6\%). OXA-48- and double carbapenemase-producers remained rare $(3.6 \%$, $6.3 \%$, respectively). Carbapenemase-producing $K$. pneumoniae isolates showed high resistance to lastline antibiotics. Gentamicin and colistin were the most active in vitro with $61.9 \%$ and $59.6 \%$ of the isolates to be inhibited at $\leq 2 \mathrm{mg} / \mathrm{L}$, followed by fosfomycin (susceptibility (S): $58.4 \%$ ) and tigecycline (S: $51.5 \%$ ). Ceftazidime/avibactam inhibited $99.6 \%$ of KPC and $100 \%$ of OXA-48-like-producing isolates, while temocillin was active against $58 \%$ of $\mathrm{KPC}$ isolates at urinary breakpoint of $\leq 32 \mathrm{mg} / L^{*}$ and only $2.7 \%$ at systemic breakpoint of $\leq 8 \mathrm{mg} / \mathrm{L}$. NDM-producing isolates belonged mainly to one clone, whereas KPC, VIM, OXA48 and double carbapenemase-producers were mainly polyclonal. Conclusions: KPC remains the predominant carbapenemase among $K$. pneumoniae in Greece, followed by NDM, whereas changing trends of resistance rates to last-line antimicrobials against carbapenemase-producing $K$. pneumoniae with the exception of ceftazidime/avibactam mandates continuing surveillance to support clinical practice.

\section{Introduction}

Hospital infections caused by carbapenem-non-susceptible Klebsiella pneumoniae constitute a worldwide problem associated with high rates of treatment failure and mortality. In Greece, carbapenem-resistance in $K$. pneumoniaeemerged in 2002 due to VIM and later, 
Carbapenem-non-susceptible Klebsiella pneumoniae clinical isolates submitted by participating hospitals, number of confirmed carbapenemase-producers and type of carbapenemase, by hospital, Greece, 2014-2016 $(\mathrm{n}=394)$

\begin{tabular}{|c|c|c|c|c|c|c|c|c|c|c|c|c|c|}
\hline \multirow{3}{*}{ Participating hospitals } & \multirow{3}{*}{$\begin{array}{l}\text { Carbapenem-non- } \\
\text { susceptible } K . \text { pneumonia } \\
\text { isolates submitted } \\
\text { n } \\
\end{array}$} & \multicolumn{10}{|c|}{ Confirmed carbapenemase-producing $K$. pneumoniae isolates } & \multirow{2}{*}{\multicolumn{2}{|c|}{$\begin{array}{l}\text { Other } \\
\text { mechanism of } \\
\text { carbapenem } \\
\text { resistance }\end{array}$}} \\
\hline & & \multicolumn{2}{|l|}{ KPC } & \multicolumn{2}{|c|}{ NDM } & \multicolumn{2}{|c|}{ VIM } & \multicolumn{2}{|c|}{$\begin{array}{l}\text { OXA-48- } \\
\text { like }\end{array}$} & \multicolumn{2}{|c|}{$\begin{array}{l}\text { KPC + VIM or } \\
\text { other dual } \\
\text { combination }\end{array}$} & & \\
\hline & & $n$ & $\%$ & $\mathrm{n}$ & $\%$ & $\mathrm{n}$ & $\%$ & n & $\%$ & $\mathrm{n}$ & $\%$ & n & $\%$ \\
\hline Athens metropolitan area & 167 & 103 & 61.7 & 25 & 15.0 & 20 & 12.0 & 12 & 7.2 & 7 & 4.2 & o & 0.0 \\
\hline $\begin{array}{l}\text { University General } \\
\text { Hospital Attikon }{ }^{\mathrm{a}}\end{array}$ & 32 & 15 & 46.9 & 10 & 31.2 & 1 & 3.1 & 4 & 12.5 & 2 & 6.2 & 0 & 0.0 \\
\hline $\begin{array}{l}\text { General Hospital of Athens } \\
\text { 'G. Gennimatas' }\end{array}$ & 25 & 19 & 76.0 & 2 & 8.0 & 0 & 0.0 & 4 & 16.0 & 0 & 0.0 & 0 & 0.0 \\
\hline KAT Hospital & 48 & 34 & 70.8 & 2 & 4.2 & 8 & 16.7 & 1 & 2.1 & $3^{b}$ & 6.2 & 0 & 0.0 \\
\hline $\begin{array}{l}\text { Hippokration Athens } \\
\text { General Hospital }\end{array}$ & 20 & 13 & 65.0 & 4 & 20.0 & 2 & 10.0 & 0 & 0.0 & $1^{c}$ & 5.0 & 0 & 0.0 \\
\hline Athens Naval Hospital & 18 & 12 & 66.7 & 3 & 16.7 & 0 & 0.0 & 3 & 16.7 & 0 & 0.0 & 0 & 0.0 \\
\hline Thriassio General Hospital & 7 & 2 & 28.6 & 0 & 0.0 & 5 & 71.4 & 0 & 0.0 & 0 & 0.0 & 0 & 0.0 \\
\hline Hygeia General Hospital & 14 & 6 & 42.8 & 3 & 21.4 & 4 & 28.6 & 0 & 0.0 & $1^{c}$ & 7.1 & 0 & 0.0 \\
\hline St Savvas Cancer Hospital & 3 & 2 & 66.7 & 0 & 0.0 & 1 & 33.3 & 0 & 0.0 & 0 & 0.0 & 0 & 0.0 \\
\hline Thessaloniki & 90 & 60 & 66.7 & 7 & 7.8 & 5 & 5.6 & 2 & 2.2 & 13 & 14.4 & 3 & 33.3 \\
\hline $\begin{array}{l}\text { Cancer Hospital } \\
\text { of Thessaloniki } \\
\text { 'THEAGENEIO' }\end{array}$ & 43 & 30 & 69.8 & 1 & 2.3 & 4 & $9 \cdot 3$ & 0 & 0.0 & 5 & 11.6 & 3 & 7.0 \\
\hline $\begin{array}{l}\text { ‘G. Papanikolaou' General } \\
\text { Hospital of Thessaloniki }\end{array}$ & 27 & 17 & 63.0 & 1 & $3 \cdot 7$ & 1 & 3.7 & 0 & 0.0 & 8 & 29.6 & 0 & 0.0 \\
\hline $\begin{array}{l}\text { General Hospital } \\
\text { of Thessaloniki ‘G. } \\
\text { Gennimatas' }\end{array}$ & 20 & 13 & 65.0 & 5 & 25.0 & 0 & 0.0 & 2 & 10.0 & 0 & 0.0 & 0 & 0.0 \\
\hline Crete & 77 & & & & & & & & & & & & \\
\hline $\begin{array}{l}\text { University Hospital of } \\
\text { Heraklion }\end{array}$ & 77 & 65 & 84.4 & 5 & 6.5 & 6 & 7.8 & 0 & 0.0 & 1 & 1.3 & 0 & 0.0 \\
\hline Central Greece & 39 & 31 & 79.5 & 1 & 2.6 & 2 & 5.1 & o & 0.0 & 4 & 10.3 & 1 & 2.6 \\
\hline $\begin{array}{l}\text { University Hospital of } \\
\text { Larissa }\end{array}$ & 20 & 18 & 90.0 & o & 0.0 & 2 & 10.0 & 0 & 0.0 & 0 & 0.0 & o & 0.0 \\
\hline General Hospital of Lamia & 19 & 13 & 68.4 & 1 & $5 \cdot 3$ & 0 & 0.0 & 0 & 0.0 & 4 & 21.1 & 1 & $5 \cdot 3$ \\
\hline Western Greece & 21 & & & & & & & & & & & & \\
\hline General Hospital of Corfu & 21 & 3 & $14 \cdot 3$ & 17 & 81.0 & 0 & 0.0 & 0 & 0.0 & 0 & 0.0 & 1 & 4.8 \\
\hline Total & 394 & 262 & 66.5 & 54 & 13.7 & 34 & 8.6 & 14 & 3.6 & 25 & 6.3 & 5 & 1.3 \\
\hline
\end{tabular}

K. pneumoniae: Klebsiella pneumoniae; KPC: Klebsiella pneumoniae carbapenemase; NDM: New Delhi metallo-beta-lactamase; VIC: Verona integron-mediated metallo-beta-lactamase.

a University Hospital Attikon contributed 32 isolates from patients hospitalised in the 4 th Department of Internal Medicine/Division of Infectious Disease.

${ }^{b}$ One isolate with KPC and OXA-48-like carbapenemase production.

c These isolates were NDM and OXA-48-like carbapenemase producers.

due to $K$. pneumoniae carbapenemase (KPC) carbapenemase production; both have become endemic $[1,2]$. The emergence of NDM-producing strains was reported in 2011 in the University Hospital of Ioannina (Epirus, Central Greece) [3]. Since then, sporadic cases have been observed in hospitals in Athens (Attica), Patras (Peloponnese, western Greece) and Larissa (Thessaly, central Greece), all belonging to multilocus sequence type (ST) 11 type [4-6]. The first OXA-48-like carbapenemase outbreak was detected in 2012 [7] and apart from single cases $[8,9]$, no major epidemics have been recorded in Greece.
According to the most recent annual surveillance report from the European Centre for Disease Prevention and Control (ECDC) [10], Greece had the highest percentage of carbapenem-resistant isolates among invasive $K$. pneumoniae in Europe but with a decreasing trend from $68.2 \%$ in 2011 to $61.9 \%$ in 2015 .

Treating carbapenem-non-susceptible $K$. pneumoniae infections is a major clinical challenge, because of the dearth of alternative drugs, often limited by poor bactericidal activity and/or high toxicity. Aminoglycosides, tigecycline, and the two revived antimicrobials, colistin and fosfomycin, are among the 
Geographical map showing the location of participating hospitals providing Klebsiella pneumoniae clinical isolates, Greece, $2014-2016(n=15)$

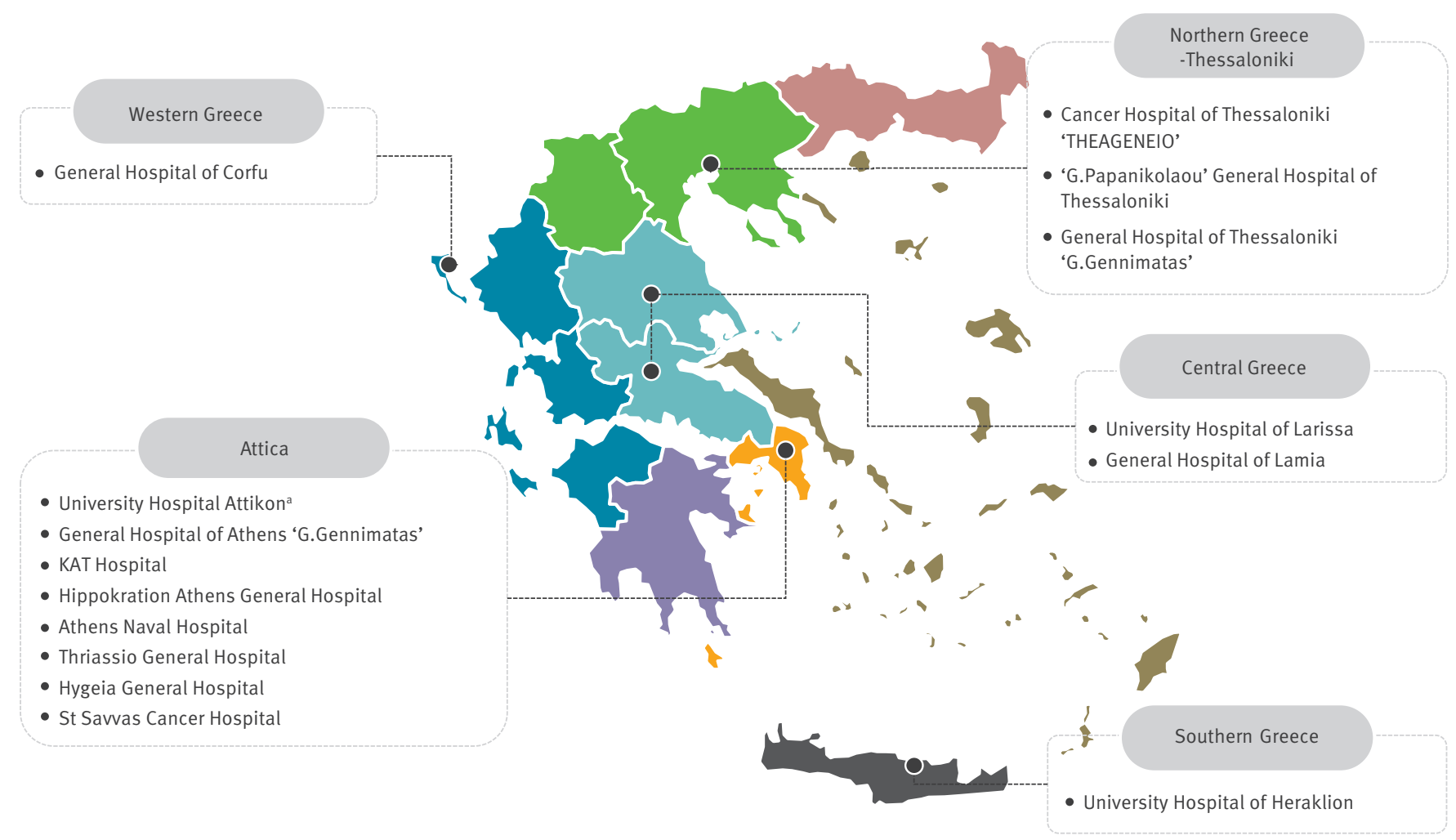

aUniversity General Hospital Attikon contributed 32 isolates from patients hospitalised in the 4th Department of Internal Medicine/Division of Infectious Diseases.

remaining options for clinicians to battle these difficult-to-treat infections [11]. Temocillin, a 6-alpha-methoxy derivative of ticarcillin, has also been suggested as a potential alternative treatment option for mild to moderately severe urinary tract infections caused by KPC-producing Enterobacteriaceae [12]. These antimicrobials in various combinations with or without a carbapenem [11,13] as well as the recently approved combination of ceftazidime/avibactam are included in therapeutic algorithms guided by the isolate MIC, the site and severity of infection, and the specific carbapenemase-encoding gene(s) [14]. It is of the utmost importance to monitor the spread of carbapenamaseproducing $K$. pneunoniae and the dissemination of various carbapenemases in Greek hospitals in order to organise strategies for infection control and the application of antibiotic stewardship.

The primary aim of this study was to evaluate the epidemiology of contemporary carbapenemase-producing $K$. pneumoniae isolated in Greek hospitals, and their susceptibilities to the antimicrobials administrated in clinical practice, i.e. meropenem, colistin, tigecycline, fosfomycin, gentamicin, temocillin and ceftazidime/avibactam. The secondary aim was to evaluate the performance of Etest and VITEK2 (both from bioMérieux, Marcy-l'Étoile, France) in comparison to the reference standard microdilution method for colistin susceptibility across these contemporary multidrugresistant isolates.

\section{Materials and methods}

\section{Setting}

Greece is a country in southern Europe with a population of ca 11 million as of 2016. Athens is the nation's capital and largest city (ca 3 million residents), followed by Thessaloniki (800,000 residents). Participating hospitals included 15 public and private tertiary- and secondary-care hospitals: eight in the Athens metropolitan area, three in Thessaloniki (northern Greece), one in Crete, the largest and most populous of the Greek islands in southern Greece, two in central Greece and one in western Greece, representing $12 \%$ of Greek hospitals.

The study was organised by the Hellenic Society of Chemotherapy in collaboration with the Infectious Diseases Laboratories of Hygeia Hospital, Athens and of the $4^{\text {th }}$ Department of Internal Medicine of 


$$
\text { | }
$$


Scattergram comparing (A) Etest minimum inhibitory concentrations and (B) VITEK2 minimum inhibitory concentrations with broth microdilution minimum inhibitory concentrations for colistin, tested against the 394 contemporary Klebsiella pneumoniae clinical isolates, Greece, 2014-2016

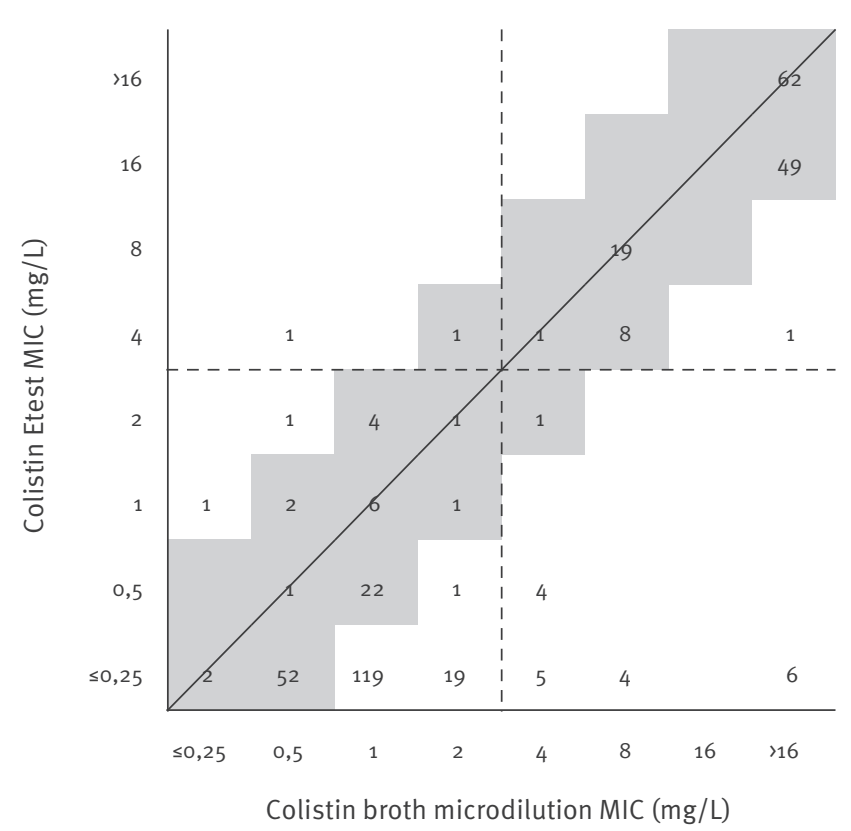

B.

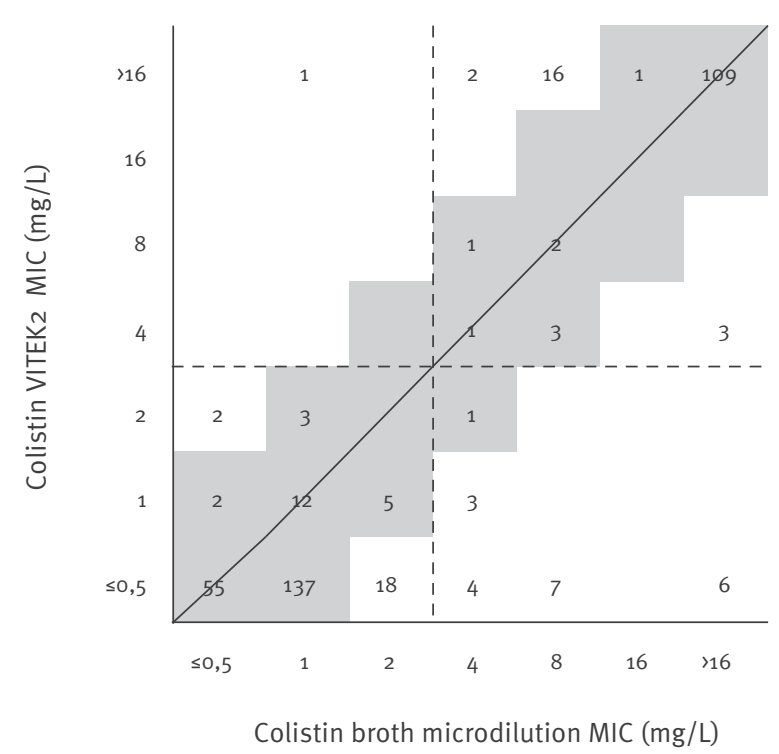

MIC: minimum inhibitory concentration.

The dashed line represents the EUCAST breakpoint for susceptibility ( $\leq 2 \mathrm{mg} / \mathrm{L})$. The diagonal line indicates absolute agreement. Shaded area indicates essential agreement. Very major errors are indicated in bold.

National and Kapodistrian University of Athens, where the susceptibility testing and molecular studies were performed. A nationwide multicentre surveillance network was formed by the microbiology laboratories of participating hospitals, which were asked to provide all consecutive single-patient $K$. pneumoniae clinical strains, isolated in a 6-month period and exhibiting non-susceptibility to any carbapenem (imipenem, meropenem or ertapenem) for further testing. These isolates were re-submitted for susceptibility testing as well as phenotypic and molecular detection of carbapenemases at a central laboratory.

Data on the source and the date of isolation as well as the initial susceptibility results at the local laboratories were also provided. All isolates were transferred to the Microbiology Laboratory of Infectious Diseases of Hygeia Hospital and kept frozen at $-80{ }^{\circ} \mathrm{C}$ until the day of testing.

\section{Phenotypic methods for detecting carbapenemase activity}

The combination disk test was used for screening carbapenemase production using meropenem $10 \mu \mathrm{g}$ disks (BIO-RAD, Marne La Coquotte, France) with or without inhibitors (phenyl boronic acid (PBA), ethylenediaminetetraacetic acid (EDTA) and cloxacillin) as recommended by the European Committee on Antimicrobial Susceptibility Testing (EUCAST) [15].

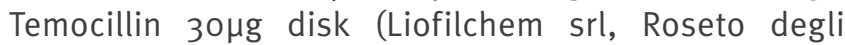
Abruzzi, Italy) was applied as a phenotypic indicator of OXA-48 production, when a tentative inhibition zone diameter $<11 \mathrm{~mm}$ was seen in the absence of synergy with PBA and EDTA. A meropenem disk with PBA and EDTA was also included to detect double carbapenemase producers (KPC and VIM), which have emerged in Greek hospitals since 2009 [16].

All isolates with negative combination disk test were further examined for carbapenemase production by the carbapenem inactivation method (CIM), a method that has $100 \%$ negative predictive value for carbapenemase production in Enterobacteriaceae [17]. To perform the CIM, a $10 \mu \mathrm{g}$ meropenem disk was immersed in a suspension of the tested isolate and incubated for a minimum of $2 \mathrm{~h}$ at $35^{\circ} \mathrm{C}$. After incubation, the disk was placed on a Mueller-Hinton agar plate inoculated with the susceptible Escherichia coli ATCC 29522 strain. If the bacterial isolate produced a carbapenemase, the meropenem in the susceptibility disk was inactivated, allowing uninhibited growth of the susceptible $E$. coli strain after $16 \mathrm{~h}$ of incubation at $35^{\circ} \mathrm{C}$. Disks 
TABLE 3A

Distribution of minimum inhibitory concentration values of carbapenem-non-susceptible Klebsiella pneumoniae isolates grouped by the mechanism of carbapenem resistance, Greece, 2014-2016 ( $\mathrm{n}=394)$

\begin{tabular}{|c|c|c|c|c|c|c|c|c|c|c|c|c|c|c|}
\hline \multirow{2}{*}{$\begin{array}{l}\text { Klebsiella pneumoniae } \\
\text { resistance genotype (n) }\end{array}$} & \multirow[t]{2}{*}{ Antibiotic } & \multicolumn{13}{|c|}{$\begin{array}{l}\text { Number of isolates inhibited at } \\
(\mathrm{mg} / \mathrm{L})\end{array}$} \\
\hline & & $\leq 0.125$ & 0.25 & 0.5 & 1 & 2 & 4 & 8 & 16 & 32 & 64 & 128 & 256 & $\geq 512$ \\
\hline \multirow{6}{*}{$\begin{array}{l}\text { All } \\
(n=394)\end{array}$} & MEM & 0 & 0 & 3 & 2 & 6 & 17 & 25 & 32 & 51 & $258^{a}$ & NA & NA & NA \\
\hline & CST (Etest) & 106 & 95 & 29 & 11 & 9 & 12 & 19 & 50 & 48 & 7 & 1 & 2 & 5 \\
\hline & CST (SensiTest) & NA & $3^{b}$ & 56 & 153 & 23 & 12 & 27 & 2 & $118^{a}$ & NA & NA & NA & NA \\
\hline & FOS & 0 & 0 & 0 & 0 & 0 & 3 & 21 & 101 & 105 & 79 & 36 & 13 & 36 \\
\hline & $\mathrm{TG}$ & 1 & 4 & 49 & 149 & 139 & 37 & 10 & 3 & 0 & 0 & 0 & 0 & 2 \\
\hline & GM & 0 & 0 & 6 & 53 & 185 & 42 & 5 & 9 & 7 & 9 & 9 & 11 & 58 \\
\hline \multirow{8}{*}{$\begin{array}{l}b l a_{\mathrm{KPC}} \\
(\mathrm{n}=\mathbf{2 6 2})\end{array}$} & MEM & 0 & 0 & 0 & 0 & 3 & 11 & 14 & 21 & 34 & $179^{a}$ & NA & NA & NA \\
\hline & CST (Etest) & 80 & 61 & 18 & 7 & 5 & 10 & 12 & 27 & 31 & 5 & 0 & 2 & 4 \\
\hline & CST (SensiTest) & NA & $2^{b}$ & 38 & 106 & 14 & 8 & 18 & 1 & $75^{\mathrm{a}}$ & NA & NA & NA & NA \\
\hline & FOS & 0 & 0 & 0 & 0 & 0 & 1 & 11 & 59 & 79 & 56 & 20 & 9 & 27 \\
\hline & TG & 0 & 3 & 35 & 98 & 96 & 24 & 4 & 2 & 0 & 0 & 0 & 0 & 0 \\
\hline & GM & 0 & 0 & 5 & 46 & 132 & 40 & 2 & 8 & 4 & 5 & 0 & 1 & 19 \\
\hline & $\mathrm{CZA}$ & 5 & 13 & 76 & 113 & 46 & 8 & 0 & 1 & 0 & 0 & 0 & o & 0 \\
\hline & TEM & 0 & 0 & 0 & 0 & 0 & 0 & 7 & 32 & 113 & 96 & 13 & 1 & 0 \\
\hline \multirow{6}{*}{$\begin{array}{l}b l a_{\mathrm{NDM}} \\
(\mathrm{n}=54)\end{array}$} & MEM & 0 & 0 & 0 & 0 & 0 & 0 & 3 & 6 & 12 & $33^{\mathrm{a}}$ & NA & NA & NA \\
\hline & CST (Etest) & 22 & 16 & 4 & 1 & 1 & 1 & 2 & 3 & 4 & 0 & 0 & 0 & 0 \\
\hline & CST (SensiTest) & NA & 0 & 8 & 29 & 5 & 2 & 3 & 0 & $7^{\mathrm{a}}$ & NA & NA & NA & NA \\
\hline & FOS & 0 & 0 & 0 & 0 & 0 & 0 & 7 & 24 & 13 & 0 & 3 & 2 & 5 \\
\hline & TG & 1 & 0 & 4 & 31 & 13 & 4 & 1 & 0 & 0 & 0 & 0 & 0 & 0 \\
\hline & GM & 0 & 0 & 0 & 1 & 22 & 0 & 0 & 1 & 0 & 2 & 5 & 6 & 17 \\
\hline \multirow{6}{*}{$\begin{array}{l}b l a_{\mathrm{vIm}} \\
(\mathrm{n}=34)\end{array}$} & MEM & 0 & 0 & 1 & 1 & 3 & 4 & 3 & 4 & 1 & $17^{\mathrm{a}}$ & NA & NA & NA \\
\hline & CST (Etest) & 5 & 10 & 5 & 2 & 1 & 1 & 4 & 2 & 2 & 1 & 0 & 0 & 1 \\
\hline & CST (SensiTest) & NA & $1^{\mathrm{b}}$ & 6 & 12 & 2 & 2 & 4 & 1 & $6^{\mathrm{a}}$ & NA & NA & NA & NA \\
\hline & FOS & 0 & 0 & 0 & 0 & 0 & 1 & 1 & 5 & 9 & 11 & 5 & 0 & 2 \\
\hline & TG & 0 & 1 & 5 & 7 & 10 & 5 & 4 & 1 & 0 & 0 & 0 & 0 & 1 \\
\hline & GM & 0 & 0 & 0 & 2 & 11 & 2 & o & 0 & 2 & 1 & 2 & 2 & 12 \\
\hline \multirow{7}{*}{$\begin{array}{l}\text { bla } a_{\text {oxA-48-like }} \\
(\mathrm{n}=14)\end{array}$} & MEM & 0 & 0 & 0 & 0 & 0 & 0 & 3 & 1 & 3 & $7^{\mathrm{a}}$ & NA & NA & NA \\
\hline & CST (Etest) & 5 & 1 & 1 & 0 & 0 & 0 & 0 & 6 & 1 & 0 & 0 & 0 & 0 \\
\hline & CST (SensiTest) & NA & 0 & 3 & 1 & 2 & 0 & 0 & 0 & $8^{\mathrm{a}}$ & NA & NA & NA & NA \\
\hline & FOS & 0 & 0 & 0 & 0 & 0 & 1 & 0 & 9 & 1 & 1 & 1 & 1 & 0 \\
\hline & TG & 0 & 0 & 1 & 9 & 2 & 0 & 1 & 0 & 0 & 0 & 0 & 0 & 1 \\
\hline & GM & 0 & 0 & 0 & 2 & 2 & 0 & 0 & 0 & 1 & 0 & 2 & 1 & 6 \\
\hline & CZA & 0 & 0 & 6 & 7 & 1 & 0 & 0 & 0 & 0 & 0 & 0 & 0 & 0 \\
\hline \multirow{6}{*}{$\begin{array}{l}b l a_{\mathrm{KPC}}+b l a_{\mathrm{vIM}} \\
(\mathbf{n}=\mathbf{2 2})\end{array}$} & MEM & 0 & 0 & 0 & 1 & 0 & 0 & 2 & 0 & 0 & $19^{\mathrm{a}}$ & NA & NA & NA \\
\hline & CST (Etest) & 0 & 2 & 0 & 0 & 0 & 0 & 1 & 10 & 8 & 0 & 1 & 0 & 0 \\
\hline & CST (SensiTest) & NA & 0 & 1 & 1 & 0 & 0 & 1 & 0 & $19^{a}$ & NA & NA & NA & NA \\
\hline & FOS & 0 & 0 & 0 & 0 & 0 & 0 & 1 & 4 & 1 & 8 & 6 & 1 & 1 \\
\hline & TG & 0 & 0 & 1 & 5 & 14 & 2 & 0 & 0 & 0 & 0 & 0 & 0 & 0 \\
\hline & GM & 0 & 0 & 0 & 1 & 18 & 0 & 3 & 0 & 0 & 0 & 0 & 0 & 0 \\
\hline
\end{tabular}

CST: colistin; CST (Etest): minimum inhibitory concentration (MIC) as determined by Etest; CST (SensiTest): MIC as determined by SensiTest Colistin; CZA: ceftazidime/avibactam; FOS: fosfomycin; GM: gentamicin; MEM: meropenem; NA: not available; TEM: temocillin; TG: tigecycline.

a Upper limit of determination, corresponds to $\geq$.

${ }^{\mathrm{b}}$ Lower limit of determination, corresponds to $\leq$.

Dark grey shading represents susceptible isolates by European Committee on Antimicrobial Susceptibility Testing (EUCAST) 2018 breakpoint. Light grey shading represents intermediate category according EUCAST 2018 breakpoint. 
Distribution of minimum inhibitory concentration values of carbapenem-non-susceptible Klebsiella pneumoniae isolates grouped by the mechanism of carbapenem resistance, Greece, 2014-2016 $(\mathrm{n}=394)$

\begin{tabular}{|c|c|c|c|c|c|c|c|c|c|c|c|c|c|c|}
\hline \multirow[t]{2}{*}{$\begin{array}{l}\text { Klebsiella pneumoniae } \\
\text { resistance genotype (n) }\end{array}$} & \multirow[t]{2}{*}{ Antibiotic } & \multicolumn{13}{|c|}{$\begin{array}{l}\text { Number of isolates inhibited at } \\
\qquad(\mathrm{mg} / \mathrm{L})\end{array}$} \\
\hline & & $\leq 0.125$ & 0.25 & 0.5 & 1 & 2 & 4 & 8 & 16 & 32 & 64 & 128 & 256 & $\geq 512$ \\
\hline \multirow{6}{*}{$\begin{array}{l}b l a_{\mathrm{NDM}}+b l a_{\text {oxA-48-like }} \\
(\mathbf{n}=\mathbf{2})\end{array}$} & MEM & 0 & 0 & 0 & 0 & 0 & 0 & 0 & 0 & 1 & $1^{\mathrm{a}}$ & NA & NA & NA \\
\hline & CST (Etest) & 1 & 1 & 0 & 0 & 0 & 0 & 0 & 0 & 0 & 0 & 0 & 0 & 0 \\
\hline & CST (SensiTest) & NA & 0 & 0 & 2 & 0 & 0 & 0 & 0 & 0 & NA & NA & NA & NA \\
\hline & FOS & 0 & 0 & 0 & 0 & 0 & 0 & 0 & 0 & 1 & 1 & 0 & 0 & 0 \\
\hline & TIG & 0 & 0 & 0 & 0 & 2 & 0 & 0 & 0 & 0 & 0 & 0 & 0 & 0 \\
\hline & GM & 0 & 0 & 0 & 0 & 0 & 0 & 0 & 0 & 0 & 1 & 0 & 0 & 1 \\
\hline \multirow{7}{*}{$\begin{array}{l}b / a_{\mathrm{KPC}}+b l a_{\text {oxA-48-like }} \\
(\mathrm{n}=1)\end{array}$} & MEM & 0 & 0 & 0 & 0 & 0 & 0 & 0 & 0 & 0 & $1^{\mathrm{a}}$ & NA & NA & NA \\
\hline & CST (Etest) & 0 & 0 & 0 & 0 & 0 & 0 & 0 & 1 & 0 & 0 & 0 & 0 & 0 \\
\hline & CST (SensiTest) & NA & 0 & 0 & 0 & 0 & 0 & 0 & 0 & $1^{\mathrm{a}}$ & NA & NA & NA & NA \\
\hline & FOS & 0 & 0 & 0 & 0 & 0 & 0 & 0 & 0 & 0 & 0 & 1 & 0 & 0 \\
\hline & TG & 0 & 0 & 0 & 0 & 0 & 1 & 0 & 0 & 0 & 0 & 0 & 0 & 0 \\
\hline & GM & 0 & 0 & 0 & 0 & 0 & 0 & 0 & 0 & 0 & 0 & 0 & 0 & 1 \\
\hline & CZA & 0 & 0 & 0 & 0 & 1 & 0 & 0 & 0 & 0 & 0 & 0 & 0 & 0 \\
\hline \multirow{8}{*}{ non- $C P(n=5)$} & MEM & 0 & 0 & 2 & 0 & 0 & 2 & 0 & 0 & 0 & $1^{\mathrm{a}}$ & NA & NA & NA \\
\hline & CST (Etest) & 1 & 2 & 0 & 0 & 0 & 0 & 0 & 0 & 1 & 1 & 0 & 0 & 0 \\
\hline & CST (SensiTest) & NA & 0 & 0 & 2 & 0 & 0 & 1 & 0 & $2^{\mathrm{a}}$ & NA & NA & NA & NA \\
\hline & FOS & 0 & 0 & 0 & 0 & 0 & 0 & 1 & 0 & 1 & 2 & 0 & 0 & 1 \\
\hline & TG & 0 & 0 & 1 & 1 & 2 & 1 & 0 & 0 & 0 & 0 & 0 & 0 & 0 \\
\hline & GM & 0 & 0 & 1 & 1 & 0 & 0 & 0 & 0 & 0 & 0 & 0 & 0 & 3 \\
\hline & CZA & 1 & 1 & 1 & 2 & 0 & 0 & 0 & 0 & 0 & 0 & 0 & 0 & 0 \\
\hline & TEM & 1 & 0 & 2 & 0 & 0 & 0 & 0 & 0 & 1 & 1 & 0 & 0 & 0 \\
\hline
\end{tabular}

CST: colistin; CST (Etest): minimum inhibitory concentration (MIC) as determined by Etest; CST (SensiTest): MIC as determined by SensiTest Colistin; CZA: ceftazidime/avibactam; FOS: fosfomycin; GM: gentamicin; MEM: meropenem; NA: not available; TEM: temocillin; TG: tigecycline.

a Upper limit of determination, corresponds to $\geq$.

b Lower limit of determination, corresponds to $\leq$.

Dark grey shading represents susceptible isolates by European Committee on Antimicrobial Susceptibility Testing (EUCAST) 2018 breakpoint. Light grey shading represents intermediate category according EUCAST 2018 breakpoint.

incubated in suspensions that did not contain any carbapenemase yielded a clear inhibition zone.

\section{Molecular analysis of carbapenemase genes}

Genotypic confirmation of carbapenemase production was conducted in all studied isolates and consisted of simplex in-house PCR assays targeting bla $a_{\mathrm{KPC}}$, bla $a_{\mathrm{NDM}}$, bla $a_{\mathrm{VIM}}$, bla $a_{\mathrm{IMP}}$ and bla $a_{\mathrm{OXA}-48}$ with specific primers and conditions (Supplement 1). Template DNA was extracted from bacteria grown in Luria Bertani broth for $18 \mathrm{~h}$ using NucleoSpin Tissue kit (MacheryNagel GmbH, Duren, Germany).

\section{Susceptibility testing}

Confirmation of the species and MIC determination of ampicillin/sulbactam, piperacillin/tazobactam, cefoxitin, ceftazidime, ceftriaxone, cefepime, aztreonam, imipenem, meropenen, amikacin, gentamicin, ciprofloxacin, levofloxacin, tigecycline, fosfomycin, colistin and trimethoprim/sulfamethoxazole was performed by VITEK2. Additionally, the MICs of meropenem, colistin, tigecycline, fosfomycin, gentamicin and temocillin were determined by Etest and ceftazidime/avibactam by Liofilchem MIC Test Strip (Liofilchem srl), according to the manufacturer's instructions. During the course of this study, the joint Clinical and Laboratory Standards Institute (CLSI)-EUCAST Polymyxin Breakpoints Working Group recommended that the reference method for colistin MIC determination be the ISO-standard broth microdilution method (20776-1) [18]. Thus, we additionally evaluated colistin MICs by the commercially available broth microdilution SensiTest Colistin (now marketed as ComASP Colistin (Liofilchem srl) according to the manufacturer's instructions.

E. coli ATCC 25922 and K. pneumoniae ATCC 700603 were included in all experiments for quality control, and all results were within accepted ranges. Results were interpreted according to EUCAST recommendations [19]. All isolates were sub-cultured twice before testing. 
Multidrug-resistant (MDR), extensively drug-resistant (XDR) and pandrug-resistant (PDR) strains were characterised as per criteria described by ECDC and the United States' Centers for Disease Control and Prevention (CDC). For this we used susceptibility data of all the above-mentioned 18 antimicrobials [20].

\section{Comparison of methods used for colistin} minimum inhibitory concentration

\section{determination}

Obtained colistin MICs were compared with the results of Etest and VITEK2 to determine essential and categorical agreement and very major error (VME) and major error (ME) rates.

Essential agreement was defined as MIC result within plus or minus one two-fold dilution from the reference result. This was adjusted for differences in the range of MICs that could be determined by the respective systems. A VME was defined when isolates were categorised as susceptible by Etest or VITEK 2 but resistant by the broth microdilution (used as the reference method). An ME was defined when isolates were resistant by Etest or VITEK2 but susceptible by the broth microdilution. Unacceptable levels were $>1.5 \%$ for VME and > $3 \%$ for $\mathrm{ME}$, as recommended in the CLSI document $\mathrm{M}_{23}-\mathrm{A}_{4}$ [21].

PCR-based screening for the plasmid-mediated colistin resistance $m c r-1 / m c r-2$ genes

Screening the entire collection of colistin-non-susceptible $K$. pneumoniae isolates $(n=159)$ for plasmidmediated colistin resistance genes, was performed by a duplex PCR protocol optimised at the Danish National Food Institute, using primers described previously from Liu et al. [22] and Xavier et al. [23] ( Supplement 1).

\section{PFGE typing}

Genetic relatedness among carbapenemase-producing $K$. pneumoniae isolates was evaluated by PFGE analysis of chromosomal restriction fragments obtained following cleavage with Spel (New England BioLabs Inc., GmbH Frankfurt am Main, Germany). A dendrogram was generated from the homology matrix with a coefficient of $1.5 \%$ using the unweighted pair-group method with arithmetic mean (UPGMA) to describe the relationships among PFGE profiles. Isolates were considered to belong to the same PFGE group if their Dice similarity index was $\geq 80 \%$ [24].

\section{Results}

Between 1 November 2014 and 30 April 2016, 15 hospitals from six Greek cities contributed a median number of 20 consecutive isolates each to the study (Figure 1 ). During the study period, 394 K. pneumoniae isolates were collected (Table 1). They originated from different clinical specimens: urine $(n=168)$, blood $(n=86)$, lower respiratory tract secretions $(n=60)$, pus $(n=55)$, cerebrospinal fluid $(n=3)$ and unknown $(n=22)$.
The combination disk test detected 389 (98.7\%) carbapenemase-producing isolates, with 263 (66.8\%) exhibiting a class A, 90 (22.8\%) a class B, 22 (5.6\%) a class $A$ and $B$ carbapenemase phenotype and 14 (3.6\%) an OXA-48-like phenotype. Five isolates (1.3\%) exhibited a negative combination disk test result and were further examined by the CIM method, which was also negative and excluded the possibility of carbapenemase production.

Carbapenemase production was confirmed in all 389 isolates (98.7\%) with a positive combination disk test, including 262 (66.5\%), 54 (13.7\%), 34 (8.6\%) and $14(3.6 \%)$ cases of bla ${ }_{\mathrm{KPC}}$, bla ${ }_{\mathrm{NDM}}$, bla $a_{\mathrm{VIM}}$ and bla $a_{\mathrm{OXA}-48}-$ like genes, respectively. Additionally, 25 (6.3\%) isolates harboured two carbapenemase genes, 22 (5.6\%) both bla $a_{\mathrm{KPC}}$ and bla $a_{\mathrm{VIM}}$, two (0.5\%) bla $\mathrm{NDM}_{\text {M }}$ and bla ${ }_{48}$-like and one isolate (0.3\%) bla $a_{\mathrm{KPC}}$ and bla $a_{\text {OXA-48 }}$-like. Isolates producing KPC or NDM along with OXA-48-like carbapenemases were falsely interpreted as only KPCor NDM-producing isolates by the combination disk test. None of the carbapenemase genes examined in this study was detected in the five isolates (1.3\%) with negative combination disk and CIM test result. Those carbapenemase-negative isolates exhibited meropenem/imipenem MICs of $0.5-4 \mathrm{mg} / \mathrm{L}$ and $0.25-0.5 \mathrm{mg} / \mathrm{L}$ respectively.

KPC enzymes, detected in $66.5 \%$ of isolates, were the most frequent carbapenemases in all but two hospitals, with a rate ranging between $14.3 \%$ and $90.0 \%$. NDM carbapenemase was the second most frequent overall $(13.7 \%)$ and was the most prominent only in one hospital (81.0\%) in western Greece (Corfu General Hospital). In four hospitals (three in Athens area and one in Thessaloniki), NDM-producers were isolated at a rate of $\geq 20 \%$ while in three hospitals (two in the Athens area and one in central Greece) none of the isolates was an NDM-producer. VIM enzymes were detected in $8.6 \%$ of the $K$. pneumoniae isolates and were the most prominent class in one hospital of Athens area. OXA48-like enzymes were detected in 14 isolates (3.6\%) in five hospitals, four in the Athens area and one in Thessaloniki.

Susceptibilities of isolates interpreted according to EUCAST breakpoints are shown in Table 2. Nineteen (4.8\%) isolates exhibited a PDR phenotype, 124 (31.5\%) exhibited an XDR phenotype, and the remaining 251 (63.7\%) an MDR phenotype. Gentamicin and colistin were the most active drugs in vitro, with $61.9 \%$ and $59.6 \%$ of the isolates found to be inhibited at $\leq 2 \mathrm{mg} / \mathrm{L}$, followed by fosfomycin (susceptibility (S): $58.4 \%$ ) and tigecycline (S: $51.5 \%)$. Fosfomycin was the most active agent against NDM (S: $81.5 \%$ ) and OXA-48 K. pneumoniae isolates (S: $78.6 \%$ ), while gentamicin was the most active against KPC plus VIM- (S: 90.1\%) and KPCproducers (S: $69.5 \%$ ). Colistin was active against $77.8 \%$ of the NDM producers and was the most active drug against the VIM-producing isolates (S: 61.8\%) (Table 
2). Co-trimoxazole (SXT) was active against $22.1 \%$ of the $K$. pneumoniae isolates.

\section{Comparison of methods used for colistin minimum inhibitory concentration determination}

Discrepancies were observed in colisin MIC values reported via the Etest vs the reference broth microdilution SensiTest Colistin (Figure 2). A trend towards lower colistin MICs was noted for Etest (Table 3), resulting in fewer colistin non-susceptible isolates (142 vs 159) and a categorical agreement of $94.7 \%$. Although there was an acceptable rate (>90\%) for the categorical agreement, a high percentage of VME (19 false susceptibles, $4.8 \%$ ) was observed, which was above the criterion of $\leq 1.5 \%$ proposed by CLSI [21]. Etest produced MICS that were $1,2,3$, and $>3 \log _{2}$ dilutions lower than those determined by SensiTest Colistin for $28.4 \%, 22.3 \%$, $20.8 \%$ and $5.8 \%$ of the isolates, respectively, resulting in a low rate of essential agreement (58.4\%).

For colistin MIC values reported via the VITEK2 system (Figure 2), the categorical and the essential agreement rates were $94.4 \%$ and $84.0 \%$ respectively; $45.2 \%$ of the isolates had MICs equal to those determined by broth microdilution, while $38.8 \%$ of the isolates had MICs that were $1 \log _{2}$ dilution higher or lower. Although categorical agreement was acceptable (> 90\%), high percentage of VME (21 false susceptible, 5.3\%) was observed, which was above the criterion of $\leq 1.5 \%$.

Temocillin MIC distributions for the 262 KPC-producing isolates tested are shown in Table 3. Overall, only seven of the KPC-producing isolates $(2.7 \%)$ were susceptible to temocillin, according to the British Society for Antimicrobial Chemotherapy (BSAC) systemic breakpoint (MICs $\leq 8 \mathrm{mg} / \mathrm{L})$. At the higher BSAC urinary breakpoint ( $\leq 32 \mathrm{mg} / \mathrm{L}), 152(58.0 \%)$ isolates were susceptible to temocillin (Table 2). However, 110 isolates (42.0\%) remained non-susceptible with MICs > 32 $\mathrm{mg} / \mathrm{L}$. On the contrary, ceftazidime-avibactam was very active against isolates carrying $b a_{\mathrm{KPC}}$, or bla $a_{\mathrm{OXA}-4 \text {-like }}$ or both ( $\mathrm{MIC}_{50} /{ }_{90}, 0.5 / 2 \mathrm{mg} / \mathrm{L}$; Table 1 ). This combination inhibited $99.6 \%$ of the non-MBL isolates at the breakpoint of $\leq 8 \mathrm{mg} / \mathrm{L}$.

Meropenem retained activity against $2.8 \%$ of $K$. pneumoniae isolates (MICs $\leq 2 \mathrm{mg} / \mathrm{L})$, while $18.8 \%(\mathrm{n}=74)$ exhibited moderately elevated carbapenem MICs (4-16 mg/L). Discrepancies were observed in meropenem MIC values reported via the VITEK2 system vs the Etest. Forty-five isolates (11.4\%), all carbapenemase producers, exhibiting MICs $0.5-8 \mathrm{mg} / \mathrm{L}$ by Etest were categorised as resistant (MIC > $8 \mathrm{mg} / \mathrm{L}$ ) to meropenem by VITEK2. Additionally, 31 isolates (7.9\%) with an Etest MIC of $16 \mathrm{mg} / \mathrm{L}$, were categorised as resistant with a MIC > 8mg/L by VITEK2. These strains were reported as non-susceptible to carbapenems by the microbiology laboratories applying VITEK2 [25].

\section{PFGE typing}

PFGE genotyping revealed a prevalent PFGE profile among KPC-producing $K$. pneumoniae, designated A, detected in $49.2 \%$ of the isolates, consisting of several variants ( $A_{1}$ to $\left.A 9\right)$, all of which were detected in more than one centre. A second PFGE profile, designated $B$, consisting of three variants ( $B_{1}$ to $B_{3}$ ), included 15 isolates, while a third PFGE profile, designated $\mathrm{C}$, was detected only in two centres in Thessaloniki area, and consisted of two variants ( $\mathrm{C}_{1}$ and $\mathrm{C}_{2}$ ), including 12 and three isolates, respectively. Twenty-four more profiles each included $2-10$ isolates.

The 34 VIM-, the 22 KPC- and VIM- and the 14 OXA-48like - producing $K$. pneumoniae isolates were multiclonal, with 17,3 and 7 clones respectively, differing between hospitals and different clones present within a single hospital, with no particular clone prevailing.

On the contrary, PFGE genotyping of NDM-producing isolates demonstrated great genetic similarity in the 52 (96.3\%) isolates (dominant clone A), consisting of three main variants ( $A_{1}$ to $\left.A_{3}\right)$, two of which were detected in more than one centre. Additionally, two isolates, each with distinct PFGE profile, were also detected (1.9\%). Moreover, two isolates carrying both $b a_{\mathrm{NDM}}$ and $b l a_{\mathrm{OXA} \text { - }}$

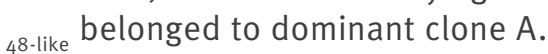

\section{Discussion}

This nationwide, multicentre study showed that among 394 contemporary carbapenem-non-susceptible consecutive $K$. pneunoniae isolates collected at 15 hospitals from six cities in Greece, KPC remains the most prevalent carbapenemase (66.5\%), followed by NDM (13.7\%). VIM has declined, from being the exclusive carbapenemase detected in Greece until 2006 [1], to $8.4 \%$ in 2016 and is followed by double carbapenemase production (5.6\%) and OXA-48 (3.6\%). These findings are in accordance with those from the European Survey on Carbapenemase-Producing Enterobacteriaceae (EuSCAPE) project, conducted between November 2013 and April 2014, in which Grundmann et al. reported for the first time that NDM was the second-ranking carbapenemase in Greece [26]. In a previous published nationwide surveillance study from January 2011 to June 2012, among 119 Greek hospitals, the prevalent mechanism of carbapenem resistance in $K$. pneumoniae isolates was KPC (82.6\%), followed by VIM (9.7\%), while the concurrent production of KPC and VIM was noted in $7.7 \%$ of the isolates [27].

The Greek situation differs greatly from other European countries such as Spain, France, Germany, Turkey, Romania and Belgium, where OXA-48, most often linked with community-onset healthcare associated sources, is the most frequently encountered carbapenemase [26,28-31].

In terms of molecular typing, we identified $\geq 80 \%$ similarity among $96.3 \%$ of the NDM isolates, indicative 
of a single clone, expanding within and between 12 hospitals from different regions in Greece. This is in accordance with previous reports that one ST11 bla $a_{\mathrm{NDM}}$ positive clone has quickly and successfully established its presence in four other Greek hospitals [3,4,32].

In the nationwide surveillance reported by Maltezou et al., it was observed that colistin, gentamicin and tigecycline resistance among carbapenem-resistant $K$.pneumoniae was $23.0 \%, 19.7 \%$ and $22.4 \%$, respectively [27]. In our collection of isolates, colistin non-susceptibility rate mounted to $40.4 \%$, and is probably related to chromosomal mutations, as no $\mathrm{mcr}$-1-positive isolates were identified among the non-susceptible strains tested. The referred mean colistin non-susceptibility rate is much higher compared to the $28.3 \%$ observed by the EuSCAPE project among 36 participating countries [26]. This increasing trend could be ascribed to increased consumption of colistin in Greece from 0.0024 defined daily doses (DDD) per 1,000 patient-days (in 2001) to 0.0480 (in 2008) and in 0.0949 (in 2015) according to data available from the European Surveillance of Antimicrobial Consumption Network (ESAC-NET) database [33]. On the other hand, the suboptimal methods for susceptibility testing used in previous studies, which underestimated the level of resistance, could be an additional explanation for the increased rate of colistin resistance in our study (method-dependent resistance). It is of great importance to emphasise that antimicrobial susceptibility testing of colistin has been fraught with difficulties, which resulted in the necessity for the recently issued recommendations from CLSI and EUCAST [15]. Although not initially included in the aim of our study, colistin susceptibility testing with the gold-standard method of broth microdilution was performed and evaluation of performance of Etest and VITEK2 was also conducted and concluded in high rates of VME across both methods; both Etest and VITEK2 underestimated colistin MIC values and understated resistance $[15,34,35]$.

Antimicrobial susceptibility testing confirmed MICs to temocillin > $8 \mathrm{mg} / \mathrm{L}$ in nearly all KPC-producing $K$. pneumoniaeisolates, precluding its use for systemic infections. However, when applying the urinary tract infection breakpoint of $\leq 32 \mathrm{mg} / \mathrm{L}, 58.4 \%$ of $\mathrm{KPC}$ producers were susceptible, suggesting a possible role of temocillin in the treatment of urinary tract infections due to those isolates. Conversely, a variable susceptibility profile was observed for fosfomycin, depending on enzyme type production and ranged from $27.3 \%$ to $81.5 \%$. In a systemic review, the reported susceptibility of fosfomycin in $K$. pneumomiae, mainly including KPC-producing $K$. pneumoniae ranged between $39.2 \%$ and $100 \%$ [36]. Intravenous fosfomycin, a revived antibiotic, has been used in carbapenem resistant Gramnegative infections, but has been associated with rapid development of resistance in vitro, and therefore monotherapy should be avoided [11]. Consequently, carbepenem- and colistin-sparing regimens for infections with MDR and XDR pathogens are welcome in settings with high resistance rates to last-line antimicrobials.

The combination ceftazidime/avibactam was active against $99.6 \%$ of KPC/OXA-48 K. pneumoniae isolates. Only one KPC-producing isolate was non-susceptible (MIC $16 \mathrm{mg} / \mathrm{L}$ ), for which further characterisation is necessary to confirm the mechanism of resistance. Production of KPC represents the main mechanism of carbapenem resistance among $K$. pneumoniae in Greek hospitals and this carbapenemase appears to be very well inhibited by avibactam. Thus the recently introduced combination represents a valuable treatment option for infections caused by KPC-producing K. pneumoniae isolates, including those caused by organisms resistant to most other antimicrobial agents, but knowledge of the underlying mechanism of resistance is required for its use [14].

In our collection, $2.8 \%$ of the $K$. pneumoniae isolates were susceptible to meropenem (MIC $\leq 2 \mathrm{mg} / \mathrm{L}), 10.7 \%$ were intermediately resistant (MIC 4-8 mg/L), while another $8.1 \%$ exhibited an MIC of $16 \mathrm{mg} / \mathrm{L}$. For these isolates $(21.6 \%)$, carbapenems are likely to maintain their clinical efficacy, especially in combination regimens [25,37]. In countries with a high incidence of carbapenemase-producing Enterobacteriaceae, like Greece, it seems imperative for an accurate method for meropenem MIC determination to be performed besides automated methods, as MIC values are important predictors of carbapenem effectiveness. The major limitation of the present multicentre surveillance study concerns the limited number of the participating Greek hospitals (12\%), mainly attributed to financial limitations. Nevertheless, public and private tertiary- and secondary-care hospitals located in all major Greek cities were purposely included in order to represent the current situation in Greece. On the other hand, advantages of the present study include the simultaneous evaluation of the in vitro activity of all clinically relevant antimicrobials, the comparison of performance of three different methods for colistin susceptibility testing, the number of contemporary clinical isolates upon which obtained results were based, and most importantly, the use of a central laboratory for the microbiological studies, so that results could be comparable.

To conclude, this study demonstrated that in nosocomial carbapenem-non-susceptible strains of $K$. pneumoniaeconsecutively collected from Greek hospitals: (i) $\mathrm{KPC}$ enzyme remained the predominant carbapenemase; (ii) NDM, surprisingly when compared with results from previous years, was the second-ranking carbapenemase; (iii) NDM-harbouring isolates belonged to a single clone, while the KPC, VIM, double carbapenemase producing and OXA-48 isolates were polyclonal; (iv) colistin MIC determination by broth microdilution is imperative and (v) the steeply increasing resistance to last-line antimicrobials such as colistin in Greece mandates the necessity of continuous 
surveillance as well as the application of strict contact precautions along with antimicrobial stewardship.

\section{${ }^{\star}$ Erratum}

Due to an error, $\leq 32 \mathrm{mg} / \mathrm{L}$ was mistakenly reported as $\leq$ 2mg/L. This was corrected on 10 August 2018.

\section{Acknowledgements}

The Authors would like to acknowledge Konstantina Nafplioti (PhD student) and Panagiota Adamou (technician) from the Infectious Diseases Laboratory of the 4 th Department of Internal Medicine in 'Attikon University Hospital', for the molecular testing of the isolates, as well as all the staff from Microbiology Departments of the 15 participating hospitals, for providing the test isolates. The study collaborators were: Viktoria Eirini Mauromanolaki, Department of Clinical Bacteriology, Parasitology, Zoonoses and Geographical Medicine, University Hospital of Heraklion, Heraklion; Sofia Tsiplakou, Microbiology Department, KAT Hospital, Athens; loanna Diamanti, Cancer Hospital of Thessaloniki 'THEAGENEIO', Thessaloniki; Helen Katsifa, Microbiological Laboratories, Bacteriology Department 'G. Papanikolaou' General Hospital of Thessaloniki, Thessaloniki; Eleftheria Trikka-Graphakos, Department of Clinical Microbiology Thriassio General Hospital, Elefsina, Athens; and Angeliki Paschali, Microbiology Laboratory General Hospital of Corfu, Corfu. Some of these data were presented at the 27th European Congress of Clinical Microbiology and Infectious Diseases, 2017, Vienna, Austria (abstract Po405).

\section{Conflict of interest}

Helen Giamarellou has received funding for research by Pfizer. All other authors have no conflict of interest to declare.

\section{Authors' contributions}

Irene Galani: co-coordinator of the study, analysis of results and author of the manuscript; Ilias Karaiskos: cocoordinator of the study; Irene Karantani and Vassiliki Papoutsaki: performed experiments and recorded the data; Sofia Maraki, Vasilliki Papaioannou, Polyzo Kazila, Helen Tsorlini, Nikoletta Charalampaki, Marina Toutouza, Helen Vagiakou, Konstantinos Pappas, Anna Kyratsa, Konstantina Kontopoulou, Olga Legga, Efthymia Petinaki, Helen Papadogeorgaki, Efrosini Chinou and study collaborators: screening of isolates and collection of data at local participating laboratories; Maria Souli: review of the manuscript; Helen Giamarellou: coordinator of the study and review of the manuscript.

\section{References}

1. Vatopoulos A. High rates of metallo-beta-lactamase-producing Klebsiella pneumoniae in Greece--a review of the current evidence. Euro Surveill. 2008;13(4):8023. https://doi. org/10.2807/ese.13.04.08023-en PMID: 18445397

2. Giakkoupi P, Papagiannitsis CC, Miriagou V, Pappa O, Polemis $\mathrm{M}$, Tryfinopoulou K, et al. An update of the evolving epidemic of blaKPC-2-carrying Klebsiella pneumoniae in Greece (200910). J Antimicrob Chemother. 2011;66(7):1510-3. https://doi. org/10.1093/jac/dkr166 PMID: 21543359

3. Voulgari E, Gartzonika C, Vrioni G, Politi L, Priavali E, Levidiotou-Stefanou S, et al. The Balkan region: NDM-1producing Klebsiella pneumoniae ST11 clonal strain causing outbreaks in Greece. J Antimicrob Chemother. 2014;69(8):20917. https://doi.org/\}\{\\\{ PMID: 24739146

4. Giakkoupi P, Tryfinopoulou K, Kontopidou F, Tsonou P, Golegou T, Souki H, et al. Emergence of NDM-producing Klebsiella pneumoniae in Greece. Diagn Microbiol Infect Dis. 2013;77(4):382-4. https://doi.org/10.1016/j. diagmicrobio.2013.09.001 PMID: 24135413

5. Spyropoulou A, Bartzavali C, Vamvakopoulou S, Marangos M, Anastassiou ED, Spiliopoulou I, et al. The first NDM metallo$\beta$-lactamase producing Klebsiella pneumoniae isolate in a University Hospital of Southwestern Greece. J Chemother. 2016;28(4):350-1. https://doi.org/10.1179/197394781 5Y.0000000003 PMID: 25671611

6. Papagiannitsis CC, Malli E, Florou Z, Sarrou S, Hrabak J, Mantzarlis K, et al. Emergence of sequence type 11 Klebsiella pneumoniae coproducing NDM-1 and VIM-1 metallo- $\beta$-lactamases in a Greek hospital. Diagn Microbiol Infect Dis. 2017;87(3):295-7. https://doi.org/10.1016/j. diagmicrobio.2016.12.008 PMID: 27993422

7. Voulgari E, Zarkotou O, Ranellou K, Karageorgopoulos DE, Vrioni G, Mamali V, et al. Outbreak of OXA-48 carbapenemaseproducing Klebsiella pneumoniae in Greece involving an ST11 clone. J Antimicrob Chemother. 2013;68(1):84-8. https://doi. org/\}\{\\\{ PMID: 22945916

8. Voulgari E, Poulou A, Dimitroulia E, Politi L, Ranellou K, Gennimata V, et al. Emergence of OXA-162 Carbapenemaseand DHA-1 AmpC Cephalosporinase-Producing Sequence Type 11 Klebsiella pneumoniae Causing Community-Onset Infection in Greece. Antimicrob Agents Chemother. 2016;60(3):1862-4. https://doi.org/\}\{\I\}\{ PMID: 26666930

9. Galani I, Anagnostoulis G, Chatzikonstantinou M, Petrikkos G, Souli M. Emergence of Klebsiella pneumoniae co-producing OXA-48, CTX-M-15, and ArmA in Greece. Clin Microbiol Infect. 2016;22(10):898-9. https://doi.org/\}\{II\}\{ PMID: 27542333

10. European Centre for Disease Prevention and Control (ECDC). Antimicrobial resistance surveillance in Europe 2015. Annual Report of the European Antimicrobial Resistance Surveillance Network (EARS-Net). Stockholm: ECDC; 2017. Available from: https://ecdc.europa.eu/sites/portal/files/media/ en/publications/Publications/antimicrobial-resistanceeurope-2015.pdf

11. Karaiskos I, Giamarellou H. Multidrug-resistant and extensively drug-resistant Gram-negative pathogens: current and emerging therapeutic approaches. Expert Opin Pharmacother. 2014;15(10):1351-70. https://doi.org/10.1517/14656566.2014.91 4172 PMID: 24766095

12. Adams-Haduch JM, Potoski BA, Sidjabat HE, Paterson DL, Doi Y. Activity of temocillin against KPC-producing Klebsiella pneumoniae and Escherichia coli. Antimicrob Agents Chemother. 2009;53(6):2700-1. https://doi.org/10.1128/ AAC.00290-09 PMID: 19332667

13. Trecarichi EM, Tumbarello M. Therapeutic options for carbapenem-resistant Enterobacteriaceae infections. Virulence. 2017;8(4):470-84. https://doi.org/10.1080/2150559 4.2017.1292196 PMID: 28276996

14. Wright H, Bonomo RA, Paterson DL. New agents for the treatment of infections with Gram-negative bacteria: restoring the miracle or false dawn? Clin Microbiol Infect. 2017;23(10):704-12. https://doi.org/10.1016/j.cmi.2017.09.001 PMID: 28893690

15. European Committee on Antimicrobial Susceptibility Testing (EUCAST). EUCAST guidelines for detection of resistance mechanisms and specific resistances of clinical and/or epidemiological importance. Version 2.0. Växjö: EUCAST; 2017. Available from: http://www.eucast.org

16. Giakkoupi P, Pappa O, Polemis M, Vatopoulos AC, Miriagou $\mathrm{V}$, Zioga A, et al. Emerging Klebsiella pneumoniae isolates coproducing KPC-2 and VIM-1 carbapenemases. Antimicrob Agents Chemother. 2009;53(9):4048-50. https://doi. org/10.1128/AAC.00690-09 PMID: 19581459

17. van der Zwaluw $\mathrm{K}$, de Haan A, Pluister GN, Bootsma HJ, de Neeling AJ, Schouls LM. The carbapenem inactivation method (CIM), a simple and low-cost alternative for the Carba NP test to assess phenotypic carbapenemase activity in gram-negative rods. PLoS One. 2015;10(3):e0123690. https://doi.org/\}\{\I\}\{ PMID: 25798828

18. European Committee on Antimicrobial Susceptibility Testing (EUCAST). Recommendations for MIC determination of colistin (polymyxin E) as recommended by the joint CLSI-EUCAST Polymyxin Breakpoints Working Group. Växjö: EUCAST; 2016. Available from: http://www.eucast.org/fileadmin/src/media/ PDFs/EUCAST_files/General_documents/Recommendations for_MIC_determination_of_colistin_March_2016.pdf

19. European Committee on Antimicrobial Susceptibility Testing (EUCAST). Clinical breakpoints version 7.1. In European 
Committee on Antimicrobial Susceptibility Testing. Växjö: EUCAST; 2017. Available from: http://www.eucast.org

20. Magiorakos AP, Srinivasan A, Carey RB, Carmeli Y, Falagas ME, Giske CG, et al. Multidrug-resistant, extensively drugresistant and pandrug-resistant bacteria: an international expert proposal for interim standard definitions for acquired resistance. Clin Microbiol Infect. 2012;18(3):268-81. https:// doi.org/10.1111/j.1469-0691.2011.03570.x PMID: 21793988

21. Clinical and Laboratory Standards Institute (CLSI). Development of In Vitro Susceptibility Testing Criteria and Quality Control Parameters. 4th ed. CLSI guideline M23. Wayne, PA: CLSI; 2016.

22. Liu YY, Wang Y, Walsh TR, Yi LX, Zhang R, Spencer J, et al. Emergence of plasmid-mediated colistin resistance mechanism MCR-1 in animals and human beings in China: a microbiological and molecular biological study. Lancet Infect Dis. 2016;16(2):161-8. https://doi.org/10.1016/S14733099(15)00424-7 PMID: 26603172

23. Xavier BB, Lammens C, Ruhal R, Kumar-Singh S, Butaye $P$, Goossens $H$, et al. Identification of a novel plasmid-mediated colistin-resistance gene, mcr-2, in Escherichia coli, Belgium, June 2016. Euro Surveill. 2016;21(27):30280. https://doi. org/10.2807/1560-7917.ES.2016.21.27.30280 PMID: 27416987

24. van Belkum A, Tassios PT, Dijkshoorn L, Haeggman S, Cookson B, Fry NK, et al. European Society of Clinical Microbiology and Infectious Diseases (ESCMID) Study Group on Epidemiological Markers (ESGEM). Guidelines for the validation and application of typing methods for use in bacterial epidemiology. Clin Microbiol Infect. 2007;13(Suppl 3):1-46. https://doi. org/10.1111/j.1469-0691.2007.01786.x PMID: 17716294

25. Petrosillo N, Giannella M, Lewis R, Viale P. Treatment of carbapenem-resistant Klebsiella pneumoniae: the state of the art. Expert Rev Anti Infect Ther. 2013;11(2):159-77. https://doi. org/10.1586/eri.12.162 PMID: 23409822

26. Grundmann H, Glasner C, Albiger B, Aanensen DM, Tomlinson CT, Andrasević AT, et al. Occurrence of carbapenemaseproducing Klebsiella pneumoniae and Escherichia coli in the European survey of carbapenemase-producing Enterobacteriaceae (EUSCAPE): a prospective, multinational study. Lancet Infect Dis. 2017;17(2):153-63. https://doi. org/10.1016/S1473-3099(16)30257-2 PMID: 27866944

27. Maltezou HC, Kontopidou F, Dedoukou X, Katerelos P, Gourgoulis GM, Tsonou P, et al. . Action Plan to combat infections due to carbapenem-resistant, Gram-negative pathogens in acute-care hospitals in Greece. J Glob Antimicrob Resist. 2014;2(1):11-6. https://doi.org/10.1016/j. jgar.2013.06.002 PMID: 27873631

28. Palacios-Baena ZR, Oteo J, Conejo C, Larrosa MN, Bou G, Fernández-Martínez $M$, et al. Comprehensive clinical and epidemiological assessment of colonisation and infection due to carbapenemase-producing Enterobacteriaceae in Spain. J Infect. 2016;72(2):152-60. https://doi.org/10.1016/j. jinf.2015.10.008 PMID: 26546855

29. Kaase M, Schimanski S, Schiller R, Beyreiß B, Thürmer A, Steinmann J, et al. Multicentre investigation of carbapenemase-producing Escherichia coli and Klebsiella pneumoniae in German hospitals. Int J Med Microbiol. 2016;306(6):415-20. https://doi.org/10.1016/j. ijmm.2016.05.009 PMID: 27237423

30. Huang TD, Bogaerts P, Berhin C, Hoebeke M, Bauraing C, Glupczynski Yon behalf of a multicentre study group. Increasing proportion of carbapenemase-producing Enterobacteriaceae and emergence of a MCR-1 producer through a multicentric study among hospital-based and private laboratories in Belgium from September to November 2015. Euro Surveill. 2017;22(19):30530. https://doi. org/10.2807/1560-7917.ES.2017.22.19.30530 PMID: 28537547

31. Dortet L, Cuzon G, Ponties V, Nordmann P. Trends in carbapenemase-producing Enterobacteriaceae, France, 2012 to 2014. Euro Surveill. 2017;22(6):30461. https://doi. org/10.2807/1560-7917.ES.2017.22.6.30461 PMID: 28205502

32. Spyropoulou A, Papadimitriou-Olivgeris M, Bartzavali C, Vamvakopoulou S, Marangos M, Spiliopoulou I, et al. A tenyear surveillance study of carbapenemase-producing Klebsiella pneumoniae in a tertiary care Greek university hospital: predominance of KPC- over VIM- or NDM-producing isolates. J Med Microbiol. 2016b;65(3):240-6. https://doi.org/10.1099/ jmm.0.000217 PMID: 26698320

33. European Centre for Disease Prevention and Control (ECDC). Antimicrobial consumption. Surveillance and disease data. Antimicrobial consumption database. Trend by country. Stockholm: ECDC. [Accessed: November 2017]. Available from: http://ecdc.europa.eu/en/healthtopics/antimicrobialresistance-and-consumption/antimicrobial-consumption/esacnet-database/Pages/trend-consumption-by-country.aspx

34. Tan TY, Ng SY. Comparison of Etest, Vitek and agar dilution for susceptibility testing of colistin. Clin Microbiol
Infect. 2007;13(5):541-4. https://doi.org/10.1111/j.14690691.2007.01708.x PMID: 17371537

35. Hindler JA, Humphries RM. Colistin MIC variability by method for contemporary clinical isolates of multidrug-resistant Gramnegative bacilli. J Clin Microbiol. 2013;51(6):1678-84. https:// doi.org/10.1128/JCM.03385-12 PMID: 23486719

36. Vardakas KZ, Legakis NJ, Triarides N, Falagas ME. Susceptibility of contemporary isolates to fosfomycin: a systematic review of the literature. Int J Antimicrob Agents. 2016;47(4):269-85. https://doi.org/10.1016/j. ijantimicag.2016.02.001 PMID: 27013000

37. Tzouvelekis LS, Markogiannakis A, Piperaki E, Souli M, Daikos GL. Treating infections caused by carbapenemase-producing Enterobacteriaceae. Clin Microbiol Infect. 2014;20(9):862-72. https://doi.org/10.1111/1469-0691.12697 PMID: 24890393

\section{License and copyright}

This is an open-access article distributed under the terms of the Creative Commons Attribution (CC BY 4.0) Licence. You may share and adapt the material, but must give appropriate credit to the source, provide a link to the licence, and indicate if changes were made.

This article is copyright of the authors, 2018. 